\title{
A Comparison of the Eigenvalue Method and The Geometric Mean Procedure for Ratio Scaling
}

\author{
David V. Budescu \\ University of Haifa \\ Rami Zwick \\ University of North Carolina at Chapel Hill \\ Amnon Rapoport \\ University of Haifa
}

\begin{abstract}
This article evaluates and compares the performance of two ratio scaling methods, the eigenvalue method proposed by Saaty $(1977,1980)$ and the geometric mean procedure advocated by Williams and Crawford (1980), given random data. The two methods were examined in a series of monte carlo simulations for two response methods (direct estimation and constant sum) and various numbers of stimuli and response scales.
\end{abstract}

\begin{abstract}
The sampling distributions of the measures of consistency of the two methods were tabulated, rules for detecting and rejecting inconsistent respondents are outlined, and approximation formulas for other designs are derived. Overall, there was a high level of agreement and correspondence between the results from the two scaling techniques even when the data were random.
\end{abstract}

The development of analytical procedures and experimental techniques for constructing ratio measurement scales has long been a major topic and important challenge in psychophysics and other areas of psychology. The interest in ratio scales is obviously related to their high level of invariance (unique up to a power transformation) and the associated statistical operations they allow to be performed (Stevens, 1946). To obtain ratio scales, S. S. Stevens and his colleagues (Stevens, Mack, \& Stevens, 1960) developed the "cross modality matching" paradigm, which was originally applied to variables with a corresponding physical continuum and later generalized to social and other psychological stimuli (e.g., Lodge, 1981). Although Stevens' techniques have been widely used in numerous areas (e.g., Lodge, 1981; Stevens, 1972, 1975), their precise characterization is still a topic of debate among measurement specialists (e.g., Shepard, 1981).

Following two decades of development of ordinal level scales, which culminated in the development of nonmetric multidimensional scaling, there has recently been a renewed interest in the problem of ratio scale measurement. To a large extent, the interest in this problem is due to the development of a new ratio scaling procedure and its successful use in a variety of experimental and practical situations. The most salient characteristic of this method was apparently first discovered by Gulliksen (1959), but the procedure was fully developed, investigated, described, and applied by Saaty $(1977,1980)$.

APPLIED PSYCHOLOGICAL MEASUREMENT

Vol. 10, No. 1, March 1986, pp. 69-78

(c) Copyright 1986 Applied Psychological Measurement Inc.

0146-6216/86/010069-10\$1.75 


\section{Saaty's Eigenvalue Method (EM)}

Consider a set of $n$ stimuli with unknown scale values, $\mathbf{s}^{\prime}=\left(s_{1}, \ldots, s_{n}\right)$. Following a process of pairwise comparisons, an $n \times n$ matrix of ratios, $\mathbf{R}$, is constructed such that

$r_{i j}=s_{i} / s_{j}, \quad i, j=1, \ldots, n$.

All the entries in $\mathbb{R}$ are positive, satisfying the reciprocity condition: $r_{j i}=1 / r_{j i}$. In reality, this constraint is artificially enforced because typically only $n(n-1) / 2$ judgments are obtained and the remaining ratios are calculated by the reciprocal transformation and by assuming that $r_{i i}=1(i=1, \ldots, n)$.

Saaty's eigenvalue method (EM) procedure is based on a relatively simple and elementary property of $\mathbf{R}$-when postmultiplied by the vector of scale values, the result is a vector related to the scale values by a constant. This constant turns out to be the size of the matrix $\mathbb{R}$. Thus,

$\mathbb{R s}=n \mathbf{s}$.

This formulation suggests that the unknown scale values can be obtained by an eigenvalue-eigenvector decomposition of $\mathbf{R}$. Saaty's solution to this problem when the data are perturbed is to use the normalized right eigenvector associated with the largest eigenvalue of $\mathbf{R}$, denoted by $\lambda_{\max }$, as an estimate of the scale values.

The matrix $\mathbf{R}$ is said to be consistent if the ratios $r_{i j}$ satisfy

$r_{i j}=r_{i k} r_{k j}, \quad i, j, k=1, \ldots, n$,

for all $i, j$, and $k$. A consistent matrix is of unit rank; moreover, its only nonzero eigenvalue must be $n$ (see Equation 2). It can be shown that $\lambda_{\max }>n$ when consistency is violated. Therefore, Saaty (1977, 1980) recommended using the normalized difference

$\mu=\left(\lambda_{\max }-n\right) /(n-1)$

as a measure of inconsistency. If $\mathbb{R}$ is consistent, $\mu=0$; if not, $\mu$ is monotonically increasing in the magnitude of departure from consistency (for more details of the properties of this method, see Saaty, 1977, 1980).

Saaty's method has attracted much attention, having been successfully applied to such diverse areas as marketing (Wind \& Saaty, 1980), political science (Saaty \& Bennett, 1977), and the measurement of subjective probabilities (Yager, 1979). However, the EM has had its share of criticism. In particular, Johnson, Beine, and Wang (1979) have pointed out that for inconsistent matrices of order $n \geqslant 4$ the solution is not invariant under transposition. In other words, the right eigenvector of $\mathbb{R}^{\prime}$ (i.e., the left eigenvector of $\mathbf{R}$ ) is not necessarily the reciprocal of the right eigenvector of $\mathbb{R}^{\prime}$. This may cause difficulties in the interpretation of the scale values (see also Budescu, 1984).

\section{The Geometric Means Procedure (GM)}

Another criticism was leveled by Williams and Crawford (1980), who argued that, unlike most estimation procedures, Saaty's procedure does not optimize any of the regular loss functions, and is difficult to interpret in a statistical fashion. Moreover, it requires complex calculations since the maximum eigenvalue and the associated eigenvector are calculated by an iterative technique. Therefore, Williams and Crawford proposed estimating the scale values by the geometric means. This geometric means (GM) procedure is invariant under transposition and can be easily calculated by hand. It has long been recognized that the GMS are least squares estimates of the logarithms of the scale values (e.g., Torgerson, 1958). Williams and Crawford have shown that if the true scores are perturbed by independent lognormally distributed errors with zero mean and variance $\sigma^{2}$, the GMs are also maximum likelihood estimates of the scale values (for a theoretical justification of the lognormal distribution of errors in the judgment of 
dissimilarities, see Ramsay, 1977). To measure the inconsistency of $\mathbf{R}$, Williams and Crawford proposed using the residual mean square

$S^{2}=\sum_{i=1}^{n} \sum_{j=1}^{n}\left[\ln \left(r_{i j}\right)-\ln \left(\mathrm{GM}_{i} / \mathrm{GM}_{j}\right)\right]^{2} /(n-1)(n-2)$,

where $S^{2}$ is an estimate of $\sigma^{2} . S^{2}=0$ if $\mathbb{R}$ is consistent, and as the departure from consistency increases, $S^{2}$ increases monotonically.

\section{A Comparison Based on Perturbed Scale Values}

For a consistent judgment matrix of any order, or for any reciprocal matrix of order $n \leqslant 3$, the eigenvector of $\mathbf{R}$ (associated with $\lambda_{\max }$ ) and the vector of row GMs are equal except for a similarity transformation (e.g., Budescu, 1984). The procedures differ, however, when consistency is violated. To compare the two procedures, Williams and Crawford (1980) conducted a monte carlo study in which reciprocal matrices of order 5,7 , and 10 were perturbed by multiplying each ratio $s_{i} / s_{j}$ by errors drawn (1) from a lognormal distribution with zero mean and variance $\sigma^{2}$ or (2) from a population of ratios of uniform random variables with the same mean and variances as in the first case. Five values of $\sigma^{2}$ were examined, and each condition was repeated 1,000 times. In all cases the GM procedure outperformed Saaty's EM procedure according to both the least squares and the log-least squares criteria. Moreover, the relative advantage of the GM procedure increased with both the size of the judgment matrix $\mathbb{R}$ and the variability of error.

A particularly attractive feature of Saaty's EM is the availability of a consistency index $\mu$ (Equation 4) that separates between judgment matrices that can be maintained and interpreted and judgment matrices that must be rejected as "randomly generated data." As a rule of thumb, Saaty $(1980,1983)$ recommended using a consistency ratio denoted by C.R. and defined by

C.R. $=\mu / \mu_{n}$,

where $\mu_{n}$ is an empirical measure computed from Equation 4 for randomly generated reciprocal matrices of order $n$. The rule of thumb (presumably for 1 to 9 response scales) is to accept only judgment matrices for which C.R. $\leqslant .1$.

Although Williams and Crawford (1980) have suggested the statistic $S^{2}$ as a measure of inconsistency for the GM procedure, no corresponding rules are available for its use. More importantly, because $\mu$ and $S^{2}$ are based on two different scaling models and do not necessarily reflect the same properties of the data, it is still an open question how the two procedures compare in the null case-when the data are known to be random (rather than consistent judgments perturbed by error).

\section{Objectives}

A major purpose of this study was to contrast and evaluate the two scaling procedures in the null case and to provide guidelines for their use with real data. In this case both scaling procedures were expected to reject, by means of $\mu$ and $S^{2}$, the null hypothesis of consistency. The methodology employed in the present article is similar to that used by Saaty $(1977,1980)$ : (1) a large number of reciprocal matrices consisting of random entries were generated, (2) solutions and indices of inconsistency were calculated, and (3) the rejection rules were compared to each other.

A second purpose was to generalize the findings from the comparison of the two scaling procedures to a nonnumerical method of obtaining ratio judgments. Saaty's EM directly obtains numerical estimates of ratios in the tradition of S.S. Stevens' $(1972,1975)$ magnitude estimation. Furthermore, Saaty (1977, 
1980) strongly recommended restricting the response scale to the positive integers 1 through 9 and their reciprocals (a total of 17 possible different values). Another, equally popular, experimental procedure for eliciting ratio judgments is the "constant sum" method (e.g., Torgerson, 1958), in which a judge is required to divide a constant number of units between two stimuli in accordance to their ratio.

When the constant sum technique is employed, the derived ratio judgments are not necessarily identical to those obtained from direct ratio judgments. In particular, they are not necessarily integer values and they are likely to yield extreme ratios near the end points and more densely clustered ratios elsewhere. Because the scale values and, consequently, the two inconsistency measures are highly sensitive to the response scale (see Saaty, 1980), the two scaling procedures for the constant sum method were compared as well.

\section{Method}

A monte carlo study was conducted to compare the EM and GM procedures for scaling ratio judgments. Thirty different conditions were generated by factorially combining three independent variables:

1. The number of stimuli to be scaled: $n=4,6,8,10$, and 12 .

2. The experimental method for eliciting ratio judgments: direct estimation and constant sum.

3. The number of different responses allowed: $k=17,25$, and 99 .

The third factor of the present design reflects an assumption regarding the level of differentiation and precision which the judge may achieve when selecting a particular response. For the direct estimation method the three values of $k$ imply that the integers 1-9 (as suggested by Saaty), 1-13, 1-50, and their reciprocals are used, respectively. When the constant sum method was employed, it was assumed that the judge could divide the total number of units specified by the experimenter (or the total length of a line) in only a finite number $(k)$ of equally spaced categories. The first two levels of the third factor ( $k$ $=17,25)$ are representative of the constrained response scales used in the psychological literature. The third level $(k=99)$ was included as a reasonable approximation to the unconstrained situation in which the judge may select any real number in making his or her judgment.

For each of the 30 conditions in the 3-way factorial design described above, 1,000 matrices were generated by independently choosing $n(n-1) / 2$ uniformly distributed integers within the range of values dictated by $k$ and the method for eliciting ratio judgments. The numbers were randomly placed in the cells of an $n \times n$ matrix (but excluding the diagonal entries, which were all $1 \mathrm{~s}$ ), and assigning their reciprocal values to the corresponding transposed positions. For each matrix $\mathbf{R}_{q}(q=1, \ldots, 1,000)$ in each of the 30 conditions, four solutions were obtained: the geometric means of $\mathbf{R}_{q}$ 's rows: $\left(\mathbf{s}_{g q}\right)$, the right eigenvector of $\mathbf{R}_{q}$ : (s $\left.\mathbf{s}_{r q}\right)$, the left eigenvector of $\mathbf{R}_{q}:\left(\mathbf{s}_{l q}\right)$, and the geometric mean of the right and left eigenvectors of $\mathbf{R}_{q}:\left(\mathbf{s}_{m q}\right)$. The estimated scale values under each model and the two measures of inconsistency $\mu$ and $S_{2}$ were then computed.

\section{Results}

The sampling distributions of $\mu$ and $S^{2}$ for the direct estimation and the constant sum methods of eliciting ratio judgments were examined. The mean and median of the distribution of $\mu$ increased in both $k$ and $n$, whereas for $S^{2}$ they only increased in $k$. For a given $k$, and regardless of which method was used to elicit responses, $\mu$ increased, on the average, as the number of stimuli grew, whereas $S^{2}$ remained unchanged. Inspection of the variability and skewness measures showed that all 60 sampling distributions became more stable (i.e., less variable) and symmetric as the number of stimuli increased, and both effects were stronger for the direct estimation procedure. (Detailed statistics for these sampling distributions are reported in a technical report, see Budescu, Zwick, \& Rapoport, 1985.) 
To illustrate the increasing stability of the sampling distributions as the number of stimuli increases, Figure 1 portrays 10 sampling distributions under direct estimation for $k=17$ (the response scale advocated by Saaty, 1977, 1980) and $n=4,6,8,10$, and 12 . The right panel of the figure displays the sampling distributions for the EM and the left panel for the GM procedure. The reduction in variability and skewness is evident in Figure 1. Tables 1 and 2 present the critical values of the null sampling distributions (at $\alpha=.10, .05$, and .01 ) for testing the null hypothesis of randomly generated responses. Because both $\mu$ and $S^{2}$ vanish when the ratio judgments are consistent, the null hypothesis is rejected (and the derived scale values may be safely accepted and properly interpreted) at a given level whenever a value smaller than that listed in the appropriate table is obtained.

Because Tables 1 and 2 are most important for practical purposes, it is desirable to generalize their results to values of $n$ and $k$ other than those examined in the present study. This was achieved by fitting relatively simple multiple regression equations based on $n$, $k$, or some monotonic transformations of these two parameters. For the constant sum method, the critical values of the sampling distribution of $\mu$ are approximated by:

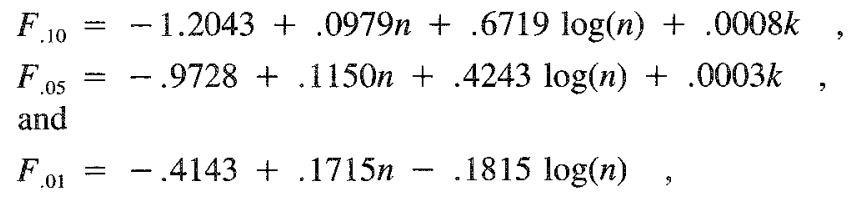

whereas the critical values for the sampling distribution of $S^{2}$ are given by:

$F_{.10}=-2.7024-.1923 n+2.9253 \log (n)+.0006 k$,

$F_{.05}=-2.6871-.1351 n+2.5866 \log (n)+.0003 k$,

and

$F_{.01}=-1.8826+.0411 n+1.3067 \log (n)+.0006 k$.

Equations 7 through 12 fit the monte carlo results extremely well; they all have adjusted squared multiple correlations of at least .99 , and all residuals are less than .085 .

For the direct estimation procedures, the critical values of $\mu$ are approximated by:

$F_{.10}=-3.1370+.9711 n-.0325 k-.0605 n^{2}+.0109 n k$,

$F_{.05}=-2.1582+.6270 n-.0355 k-.0429 n^{2}+.0144 n k$,

and

$F_{.01}=-.4382+.1744 n-.0511 k-.0155 n^{2}+.0116 n k$.

The critical values for $S^{2}$ are approximated by:

$F_{.10}=-4.5034+1.0793 n+.0688 k-.0620 n^{2}+.0062 n k-.0005 k^{2}$

$F_{.05}=-4.2697+1.0044 n+.0544 k-.0567 n^{2}+.0065 n k-.0005 k^{2}$,

and

$F_{.01}=-3.3187+.7788 n+.0190 k-.0434 n^{2}+.0077 n k-.0003 k^{2}$.

The fit of Equations 13 through 18, though very good, is not as impressive as the fit reported above for the constant sum method. All the adjusted squared multiple correlations exceed .97, but some of the residuals for the case $k=99$ seem larger than desired.

Several measures of agreement between $\mu$ and $S^{2}$ were examined and found to be quite high: the mean rank order correlation between $\mu$ and $S^{2}$ was .813 for the direct estimation and .832 for the constant sum method, and the corresponding mean percentage of agreement in rejection at $\alpha=.05$ was 81 and 86 , respectively. The two measures of inconsistency are in closer agreement for the constant sum method. 
Figure 1

Sampling Distributions of $\mu$ and $S^{2}$ under Direct Estimation for $k=17$, and $n=4,6,8,10$, and 12
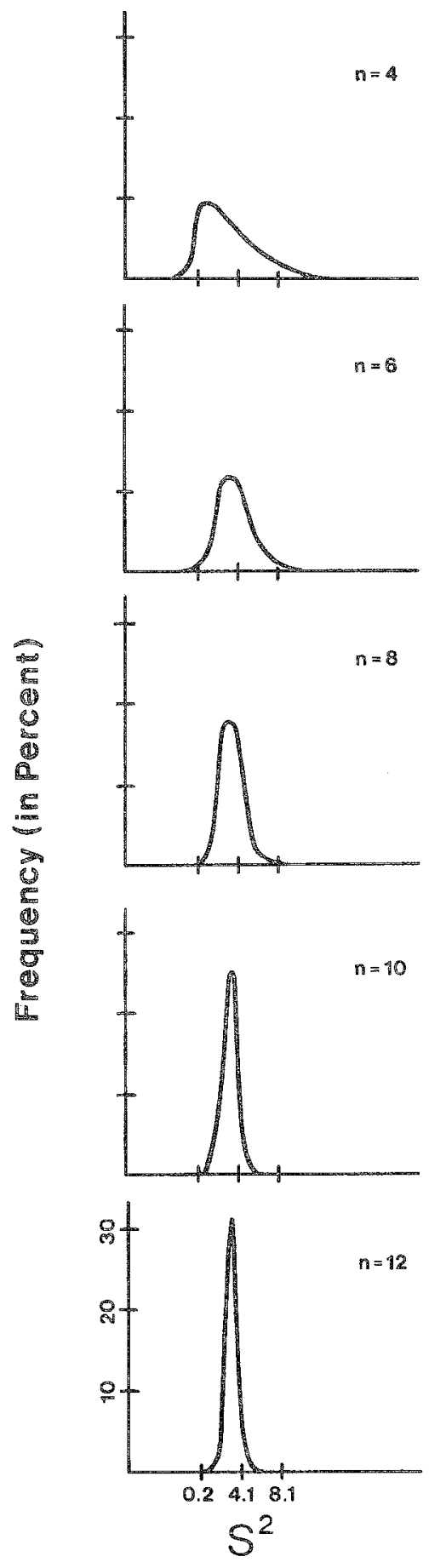
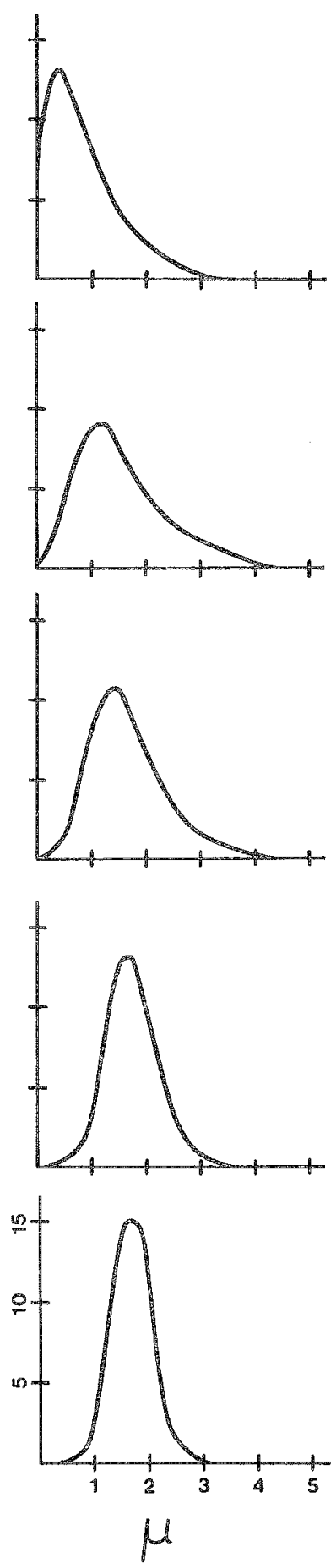
Table 1

Critical Values of the Sampling Distribution of $\mu$ and $S^{2}$ for the Direct Estimation Method

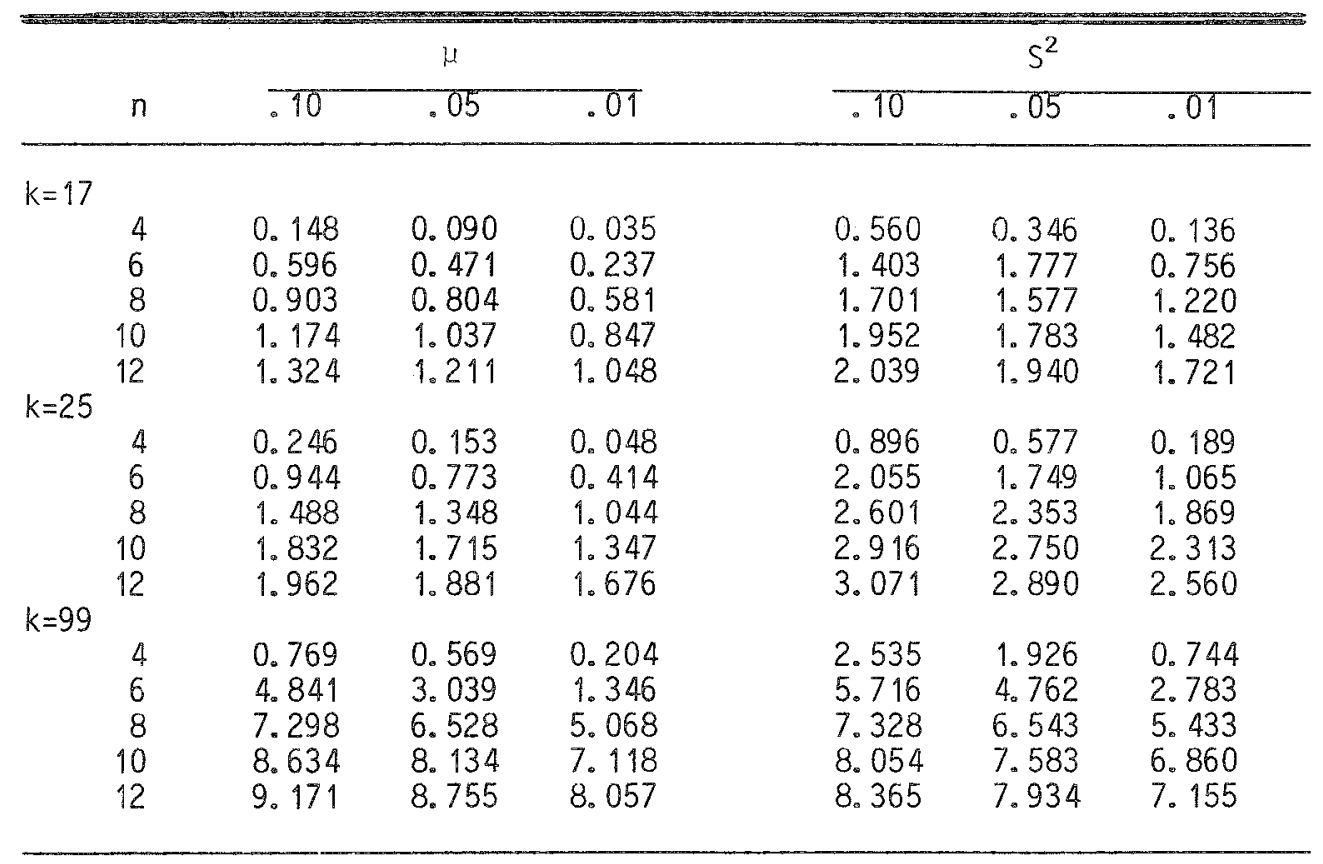

The level of agreement depends on the number of stimuli-as $n$ increases, the agreement between $\mu$ and $S^{2}$ declines.

The relations between the four solutions to the scaling problem- $-\mathbf{s}_{g}, \mathbf{s}_{l}, \mathbf{s}_{r}$, and $\mathbf{s}_{m}$-were also investigated. Table 3 presents the median Pearson product moment correlations between any two solutions across the 1,000 replications for the constant sum method. All the correlations are high, indicating that the four solutions yield similar scale values in the null case as well as in the unidimensional perturbed case (Williams \& Crawford, 1980). An examination of Table 3 reveals several systematic effects: There is a strong and consistent inverse relationship between the number of stimuli $(n)$ and the degree of association between the various pairs of solutions. Also, there are no systematic differences among the correlations due to the response scale $(k)$. Because of space considerations the correlations for the direct estimation method are not presented here. However, the same systematic effects can be observed in that case as well. In fact, the correlations between the solutions are slightly higher than under the constant sum method (see Budescu et al., 1985).

When the six correlations within a condition (row) are compared to one another, an interesting pattern emerges. In all the cases examined the lowest correlation is between the left and right eigenvectors $\left(s_{l}, s_{r}\right)$ and the highest correlation is between the geometric mean solution and the geometric mean of the two-eigenvector solution $\left(\mathbf{s}_{g}, \mathbf{s}_{m}\right)$. Interestingly, of the four solutions, only $\mathbf{s}_{g}$ and $\mathbf{s}_{m}$ are invariant under transposition of the judgment matrix $\mathbb{R}$.

\section{Discussion}

The present study provides a much needed service to those who employ ratio scaling procedures routinely in their work and who are often concerned with the reliability and consistency of their data. 
Table 2

Critical Values of the Sampling Distribution of $\mu$ and $S^{2}$ for the Constant Sum Method

\begin{tabular}{|c|c|c|c|c|c|c|c|}
\hline & \multirow[b]{2}{*}{$n$} & \multicolumn{3}{|c|}{$\mu$} & \multicolumn{3}{|c|}{$s^{2}$} \\
\hline & & .10 & .05 & .01 & .10 & .05 & .01 \\
\hline \multicolumn{8}{|l|}{$k=17$} \\
\hline & 4 & 0.164 & 0.103 & 0.036 & 0.610 & 0.393 & 0.142 \\
\hline & 6 & 0.604 & 0.487 & 0.276 & 1.410 & 1.167 & 0.675 \\
\hline & 8 & 0.999 & 0.835 & 0.534 & 1.856 & 9.604 & 1. 118 \\
\hline & 10 & 1.301 & 1.56 & 0.944 & 2.306 & 1.889 & 1.632 \\
\hline & 12 & 1.553 & 1.409 & 1. 188 & 2.210 & 2.094 & 1.851 \\
\hline$n-2 \sqrt{n}$ & 4 & 0.160 & 0.095 & 0.032 & 0.593 & 0.363 & 0.125 \\
\hline & 6 & 0.620 & 0.479 & 0.238 & 1.455 & 1.170 & 0.655 \\
\hline & 8 & 1.033 & 0.874 & 0.604 & 1,891 & 1.641 & 1.224 \\
\hline & 10 & 1.367 & 1.184 & 0.887 & 2. 151 & 1.933 & 1.563 \\
\hline \multirow{2}{*}{\multicolumn{8}{|c|}{$k=99$}} \\
\hline & & & & & & & \\
\hline & 4 & 0.166 & 0.094 & 0.034 & 0.620 & 0.359 & 0.134 \\
\hline & 6 & 0.601 & 0.455 & 0.270 & 0.270 & 1. 144 & 0.134 \\
\hline & 8 & 0.830 & 0.830 & 0.609 & 1. 871 & 1.594 & 1.245 \\
\hline & 10 & 1.456 & 1.237 & 0.952 & 2. 173 & 2.002 & 1.646 \\
\hline & 12 & 1.784 & 1.534 & 1.122 & 2. 396 & 2.202 & 1.863 \\
\hline
\end{tabular}

Tables 1 and 2 provide these researchers a sound basis for detecting inconsistent judgments and subjects. They supply decision rules for accepting or rejecting judgment matrices for both the EM and GM procedures. In the former case, it is contended that use of the traditional approach to hypothesis testing, in which the null hypothesis is rejected with a predetermined probability of Type $\mathbb{I}$ error, is superior to any rule of thumb. In the latter case, the present results fill a gap in the long line of psychometric studies on the properties of the geometric means.

Like any other simulation, the present results are limited to those combinations of parameters tested. As such, they lack the generality achievable by a theoretical development. However, the values of $n$ and $k$ employed in the present study are representative of the choices usually encountered in applications, so that many users should find the tables of this study useful. Those using different response scales and a larger number of stimuli will benefit from the approximation formulas developed for the critical points of the sampling distributions. Undoubtedly, these formulas may be further refined in similar studies covering a larger range of parameter values.

At a more general methodological level it is concluded that there is a very high level of agreement between the EM and GM procedures in the null case. This conclusion is based on (1) the high correlations between the estimated scale values, (2) the large concordance between the rank orderings of the various matrices by means of $\mu$ and $S^{2}$, and (3) the high agreement between the two rejection rules for various levels of $\alpha$. It was pointed out above that one interpretational problem of the EM stems from its lack of invariance under transposition of $\mathbb{R}$. This problem is well documented in Table 3 where the right and left eigenvectors are shown to intercorrelate lower than any other pair of solutions. This finding reinforces the warning of Johnson et al. (1979) regarding the use of the right eigenvector. It is reassuring to note in the same tables that the highest correlations are typically those between the two invariant solutions. 
Table 3

Median Correlations* Between the Four Solutions for the Constant Sum Method

\begin{tabular}{|c|c|c|c|c|c|c|c|}
\hline & $\mathrm{n}$ & $\mathrm{S}_{\mathrm{g}} \times \mathrm{s}_{\mathrm{l}}$ & $s_{g} \times s_{m}$ & $S_{g} \times S_{r}$ & $s_{e} \times s_{m}$ & $s_{e} \times s_{r}$ & $s_{m} \times s_{r}$ \\
\hline \multicolumn{8}{|l|}{$k=17$} \\
\hline & 4 & 987 & 966 & 988 & 993 & 977 & 966 \\
\hline & 6 & 917 & 970 & 913 & 945 & 789 & 958 \\
\hline & 8 & 852 & 940 & 834 & 896 & 627 & 922 \\
\hline & 10 & 814 & 922 & 791 & 881 & 538 & 892 \\
\hline & 12 & 765 & 895 & 754 & 864 & 479 & 867 \\
\hline \multicolumn{8}{|l|}{$k=25$} \\
\hline & 4 & 986 & 996 & 986 & 994 & 975 & 955 \\
\hline & 6 & 914 & 972 & 915 & 943 & 789 & 962 \\
\hline & 8 & 855 & 940 & 817 & 844 & 605 & 920 \\
\hline & 10 & 808 & 909 & 796 & 841 & 431 & 868 \\
\hline & 4 & 926 & 976 & 934 & 945 & 845 & 995 \\
\hline & 6 & 918 & 970 & 912 & 941 & 788 & 960 \\
\hline & 8 & 834 & 831 & 822 & 876 & 586 & 927 \\
\hline & 10 & 791 & 896 & 745 & 824 & 479 & 903 \\
\hline & 12 & 751 & 852 & 663 & 791 & 869 & 876 \\
\hline
\end{tabular}

* Decimal points are omitted.

The EM and GM are not the first, nor the only, methods available for scaling ratio judgments. A large number of additional techniques have been proposed, ranging from relatively simple averages (Comrey, 1950; Sjoberg, 1963) to more complicated and sophisticated methods, such as the least squares solutions (Eckart \& Young, 1936; Jensen, 1984a), weighted least squares (Chu, Kalaba, \& Spingarn, 1979), and minimum chi-square (Jensen, 1984b). Although there is a large consensus that in most cases the solutions are very similar to each other, a heated argument has developed recently in the literature regarding the "best" solution. Saaty and Vargas (1984a, 1984b) argued for the absolute superiority of EM, and Williams and Crawford (1980) and de Jong (1984) make the case for GM. Jensen's (1984b) lucid analysis of four methods according to five theoretical criteria clearly demonstrated that this dispute is futile-each method is best by at least one criterion (usually one it explicitly seeks to optimize), but neither is best by all, or most, criteria. In light of this observation and given that there is no agreement regarding the relative importance of the various criteria, it would be inappropriate to claim that the present results can cause rejection, or unconditional acceptance, of any single method. Jensen's view that multiple solutions should be reported is more acceptable. The results of this study should help evaluate scale values obtained from two of the many possible solutions.

\section{References}

Budescu, D. V. (1984). Scaling binary comparison matrices: A comment on Narasimhan's proposal and other methods. Fuzzy Sets and Systems, 14, 187-192.
Budescu, D. V., Zwick, R., \& Rapoport, A. (1985). A comparison of the analytic hierarchy process and the geometric mean procedure for ratio scaling (Report 
No. 172). Chapel Hill NC: The L. L. Thurstone Psychometric Laboratory.

Chu, A. T. W., Kalaba, R. E., \& Spingarn, K. (1979). A comparison of two methods for determining the weights of belonging to fuzzy sets. Journal of Optimization Theory and Applications, 27, 531-538.

Comrey, A. L. (1950). A proposed method for absolute ratio scaling. Psychometrika, 15, 317-325.

de Jong, P. (1984). A statistical approach to Saaty's scaling method for priorities. Journal of Mathematical Psychology, 28, 467-478.

Eckart, C. D., \& Young, G. (1936). The approximation of one matrix by another of lower rank. Psychometrika, 1, 211-217.

Gulliksen, H. (1959). Mathematical solutions for psychological problems. American Scientist, 47, 178201.

Jensen, R. E. (1984a). An alternative scaling method for priorities in hierarchical structures. Journal of Mathematical Psychology, 28, 317-332.

Jensen, R. E. (1984b). Comparisons of eigenvectors, least squares, chi square and logarithmic least squares methods of scaling a reciprocal matrix (Working $\mathrm{Pa}-$ per No. 127). San Antonio TX: School of Business, Trinity University.

Johnson, C. R., Beine, W. B., \& Wang, T. Y. (1979). Right-left asymmetry in an eigenvector ranking procedure. Journal of Mathematical Psychology, 18,6164.

Lodge, M. (1981). Magnitude scaling-Quantitative measurement of opinion. Beverly Hills CA: Sage Publications.

Ramsay, J. O. (1977). Maximum likelihood estimation in multidimensional scaling. Psychometrika, 42, 241266.

Saaty, T. L. (1977). A scaling method for priorities in hierarchical structures. Journal of Mathematical Psychology, 15, 234-281.

Saaty, T. L. (1980). The analytic hierarchy process. New York: McGraw-Hill.

Saaty, T. L. (1983). Foundations of the logical choice to harness complexity. Unpublished manuscript, School of Business, The University of Pittsburgh.

Saaty, T. L., \& Bennett, J. P. (1977). A theory of analytical hierarchies applied to political candidacy. Behavioral Science, 33, 237-245.

Saaty, T. L., \& Vargas, L. G. (1984a). Comparison of eigenvalue logarithmic least squares and least squares methods in estimating ratios. Mathematical Modelling, 5, 309-324.

Saaty, T. L., \& Vargas, L. G. (1984b). Inconsistency and rank preservation. Journal of Mathematical Psychology, 28, 205-214.
Shepard, R. N. (1981). Psychological relations and psychophysical scales: On the status of "direct" psychophysical measurement. Journal of Mathematical Psychology, 24, 21-57.

Sjoberg, L. (1963). An empirical application of a new case of the law of comparative judgment. Scandinavian Journal of Psychology, 4, 97-107.

Stevens, J. C., Mack, J. D., \& Stevens, S. S. (1960). Growth of sensation on seven continua as measured by force of handgrip. Journal of Experimental Psychology, 59, 60-67.

Stevens, S. S. (1946). On the theory of scales of measurement. Science, 103, 677-680.

Stevens, S. S. (1972). Psychophysics and social scaling. Morristown NY: General Learning Press.

Stevens, S. S. (1975). Psychophysics: Introduction to its perceptual, neural, and social prospects. New York: Wiley.

Torgerson, W. S. (1958). Theory and methods of scaling. New York: Wiley.

Williams, C., \& Crawford, G. (1980). Analysis of subjective judgment matrices (Report R-2572-AF). Santa Monica CA: Rand Corporation.

Wind, Y., \& Saaty, T. L. (1980). Marketing applications of the analytic hierarchy process. Management Science, 26, 641-658.

Yager, R. R. (1979). An eigenvalue method of obtaining subjective probabilities. Behavioral Science, $24,382-$ 387.

\section{Acknowledgments}

This research was facilitated by Contract MDA 903-83$K-0347$ from the U.S. Army Research Institute for the Behavioral and Social Sciences to the $L$. L. Thurstone Psychometric Laboratory, University of North Carolina at Chapel Hill. The views, opinions, and findings contained in this paper are those of the authors and should not be construed as an official Department of the Army position, policy, or decision. David Budescu's work was partially supported by a grant from the Faculty of Social Sciences and Mathematics at the University of Haifa. The authors thank Thomas Wallsten for his help and comments.

\section{Author's Address}

Send requests for reprints or further information to David Budescu, Department of Psychology, University of Haifa, Haifa 31999, Israel. 